\title{
ISS/SIC Donors July 2010 thru December 2010
}

The Foundation is indebted to the following colleagues for their voluntary contributions between July 1, 2010 and December 31, 2010.

Mohammed Omar Al-Asseeri, Saudi Arabia

Hazim M. Al-Haideri, Canada

Emmanuel Adoyi Ameh, Nigeria

Oluwole A. Atoyebi, Nigeria

M. Walid Baker, Sweden

Mouwafak Banno, U.S.A.

J. Alberto Cancino-Gamboa, Mexico

Edgar Cassella, Colombia

Patrick Charles Cregan, Australia

Raul Cutait, Brazil

Issam El Nakadi, Belgium

Antonio Manuel Estima-Martins, Portugal

Nobuhiro Fukunari, Japan

Atsushi Fukuuchi, Japan

Christine Gaarder, Norway

Samir Johna, U.S.A.

Abdukhakim M. Khodjibaev, Uzbekistan

Osamu Kobayashi, Japan
Alvaro Larrad Jimenez, Spain

Oladejo O. Lawal, Nigeria

Carlos Jesse Leon, Chile

Joel Umali Macalino, Philippines

Morito Maruta, Japan

Carlos Alberto G. Mesquita, Portugal

Paal Aksel Naess, Norway

Junji Okuda, Japan

George V. Oosthuizen, South Africa

Gerson A. Pereira Júnior, Brazil

Herve Probst, Switzerland

Hans-Jakob Riedtmann-Klee, Switzerland

Patricio Sanchez Fernandez, Mexico

Kate Melissa Stringer, Australia

Gerardo Rodas Thonet, Chile

Pedro Trillo Parejo, Spain

Pierre Verhaeghe, France

Hans Ernst Wagner, Switzerland

Xianzhong Wu, Republic of China

Mohamed Khaled S. Zaki, Saudi Arabia 